\title{
Flora bacteriana nasal: estudo entre médicos residentes dos Hospitais Universitários de Londrina - Paraná
}

\section{Nasal bacteriological flora: a study among medical residents of Londrina University Hospitais - Parana State - Brazil}

\author{
Zenshi Heshiki ${ }^{1}$; Regina Mariuza Borsato Quesada ${ }^{2}$; Rosana Emiko Heshiki³; \\ Daniela Matsunaga Joaquim ${ }^{3}$; Lucinda Giampietro Brandão ${ }^{3}$
}

\begin{abstract}
Resumo
As publicações sobre a flora bacteriana nasal em pessoas normais são poucas e a maioria foi realizada em países de clima temperado. O objetivo é identificar esta flora entre os médicos residentes dos hospitais da Universidade Estadual de Londrina. A coleta foi realizada com zaragatoa, e a identificação dos germes utilizou-se do método descrito anteriormente. O material consiste de 88 residentes e os resultados são apresentados em forma de tabelas. Podemos afirmar que a maioria dos médicos residentes dos Hospitais universitários de Londrina é portador de mais de 2 germes patogênicos na fossa nasal, com alta resistência aos antibióticos testados, como a penicilina (29\%), eritromicina (19\%), terramicina $12 \%$, cloranfenicol $9 \%$. Os germes apresentaram resistência menor aos antibióticos mais novos de $2 \%$ para a ciprofloxacina, oxacilina, rifampicina e a cefepime. O estafilococo coagulase negativo, considerado flora normal, também mostrou resistência elevada.
\end{abstract}

Palavras-chave: Flora bacteriana nasal, Staphylococcus aureus, Gram negativo, médicos residentes.

\begin{abstract}
There is little information about the growth of pathogenic microorganism of the nasal flora in tropical countries since most investigations were held in places with other climate. We identified the nasal flora bacteria of the medical residents of Londrina State University Hospitals. The method is the same that was described previously. The study was carried out in 88 medical residents and the results are presented in tables. It is possible confirm that the majority of the residents has 2 or more pathogenic bacteria in nasal flora with antibiotic resistance for penicillin (29\%), erythromycin (19\%), terramycin (12\%) and cloranfenicol (9\%). The resistance of the bacteria is low for the new antibiotics like ciprofloxacin, oxacilin, rifamphycin and the cephepyme.
\end{abstract}

Key-words: Nasal Bacteriological Flora; Staphylococcus aureus; Gram negative; medical residents.

\footnotetext{
${ }^{1}$ Docente Depto. CIR - Setor de Otorrinolaringologia. Av. Faria Lima, 979 - Londrina - Pr. Brasil. Tel. 43-3371-2269 Fax: 433338-2663 E-mail: zenfour@uel.br

2 Docente do Depto. MISC - Laboratório de Microbiologia

3 Alunos de pós-graduação. Mestranda.

Trabalho realizado dentro do Projeto 306.277/94 com bolsas acadêmicas UEL/CPG/CNPq/PIBIC-Universidade Estadual de Londrina-Hospital Universitário.
} 


\section{Introdução}

O conhecimento da flora nasal tem importância no contexto das infeções nosocomiais. Adquire maior importância quando se estuda a prevalência dos microorganismos da população circulante do meio hospitalar. As fossas nasais são importantes reservatórios de bactérias, e nos chamados portadores assintomáticos estas bactérias têm mostrado elevadas taxas de mutagenicidade e resistência antimicrobiana.

\section{Objetivo}

O estudo visa demonstrar as alterações quanto às características de distribuição das cepas e ao padrão de resistência antimicrobiana das bactérias albergadas nas fossas nasais de médicos residentes dos Hospitais Universitários de Londrina.

\section{Revisão Bibliográfica}

Hutchison, et al.(1957) estudaram grupos de enfermeiras coletando material das que trabalhavam de 6 a 23 semanas de treinamento. Até a $7^{\text {a }}$ semana não havia contato hospitalar e partir da $8^{\circ}$ semana esse contato aumentava até atingir contato hospitalar diário. Os autores verificaram que não existe aumento significativo na taxa de positividade nasal de estafilococos durante o período de estudo. Concluíram que $20 \%$ das enfermeiras eram portadoras persistentes, $20 \%$ eram não persistentes e $60 \%$ eram portadoras temporárias. Nestes casos, freqüentemente foram detectados o Staphylococcus aureus resistentes à penicilina.

Maxwell et al.(1969) ao estudarem Staphylococcus aureus como agente nas infeções nosocomiais, selecionaram funcionários do hospital que trabalhavam no setor cirúrgico, usando, como grupo controle, um grupo de residentes, internos e estudantes de medicina. O material coletado foi da fossa posterior de narina e realizado testes de sensibilidade à penicilina. No grupo em estudo, verificaram que $39 \%$ eram portadores intermitentes ou persistentes enquanto no grupo controle eles detectaram $31,8 \%$. Afirmam que a diferença entre os dois grupos foi insignificante. Concluíram que a taxa de presença de Staphylococcus aureus não sofreu alterações durante 4 a 5 anos, que foi o tempo de exposição hospitalar avaliado no estudo. A incidência de portadores do Staphylococcus aureus não difere da encontrada na população hospitalar em geral. $62 \%$ das pessoas que eram portadores persistentes ou intermitentes tinham organismos resistentes a penicilina, e $21 \%$ eram sensíveis à penicilina.

Rahal et al.(1970), preocupados com o aumento da incidência de infecções por Gram (-) adquiridos em hospital, os autores estudaram 70 pessoas, (15 médicos e 55 enfermeiros) tendo como controle 26 pessoas do setor administrativo. Realizaram coleta de material do nariz e garganta, no total de 192 culturas. Na equipe hospitalar encontraram $8,5 \%$ com bacilos Gram (-) como a Klebsiella sp, Proteus mirabilis e Escherichia coli. No grupo controle, 11,5\% de bacilos G (-) Klebsiella sp, Enterobacter e Escherichi coli. Investigações sorológicas e epidemiológicas evidenciam a transmissão nosocomial dos Gram (一). Vários objetos hospitalares mostraram contaminação por Gram (-) e a maioria destes com possibilidade de transmissão através de contato manual, enquanto a transmissão aérea é mínima. O trato respiratório superior de indivíduos normais são raramente colonizadas por Gram (-). Os autores mostraram que, nas pessoas saudáveis, a colonização da árvore respiratória superior por essas bactérias não é mais freqüente do que na população controle, mesmo que aquelas pessoas estejam expostas aos pacientes portadores de infecções por Gram (). Concluem que as pessoas saudáveis portadores de Gram (-) não são as responsáveis pela disseminação hospitalar. Doig, A.(1971), analisa três grupos: Grupo I (ambulatorial), no qual $26 \%$ eram portadores de Staphylococcus aureus. A presença de Staphylococcus aureus hospitalar foi estimada em $4,1 \%$. Grupo II (internados), no qual $50 \%$ eram portadores de Staphylococcus aureus, e 5,5\% eram 
portadores de Staphylococcus aureus hospitalar. Grupo III (administração), no qual 80\% eram portadores de Staphyloccus aureus, e 15\% com Staphylococcus aureus hospitalar. A incidência de feridas com Staphylococcus aureus em portadores deste agente na fossa nasal, foi semelhante naqueles pacientes não portadores.

Quanto à resistência aos antibióticos, verifica-se o seguinte:

Grupo ambulatorial: $17 \%$ foram resistentes a apenas 1 antibiótico, 15,2\% foram resistentes apenas à penicilina e $1,4 \%$ era resistente à estreptomicina ou à tetraciclina. Grupo internado: $32 \%$ Staphylococcus aureus resistente à pelo menos um antibiótico, $17 \%$ era resistente apenas à penicilina, $1 \%$ era resistente a 4 ou mais antibióticos (considerados multi resistentes MR) e $27 \%$ dos pacientes internados adquiriram resistência com a exposição ao ambiente hospitalar. Grupo da administração: 58\% resistentes à penicilina. Neste grupo não foi identificado microorganismo multiresistente

Cetin et al.(1971) estudaram 1663 estudantes universitários de vários cursos e identificaram 183 bactérias potencialmente patogênicas ou fungos de cavidade nasal. O Staphylococcus aureus isolado de fossa nasal dos estudantes de cursos de Medicina foi $11 \%$, a de Farmácia, $7 \%$, de Biologia, $16 \%$, e de Odontologia, $8 \%$. A analise da sensibilidade a antibióticos das culturas de Staphylococcus aureus mostrou resistência à penicilina em mais de $60 \%$ de todos os estudantes. Sabe-se que as bactérias potencialmente patogênicas encontradas na flora normal podem ser prejudiciais ao organismo, desde que a resistência esteja diminuída. Foi observado também que a taxa de portadores é mais alta em indivíduos com menos de 20 anos. Em outro estudo, demonstraram que as culturas das fossas nasais tem incidência de Staphylococcus aureus em 20,5\%, e a resistência à penicilina foi de $72 \%$. É de se esperar que as bactérias identificadas em estudantes de medicina sejam mais resistentes a antibióticos do que as outras pessoas da comunidade. Os resultados do trabalho, entre- tanto não mostraram essa diferença, pois a grande maioria desses estudantes é do curso básico.

Winther et al. (1974), analisaram 97 estudantes da escola de Educação Física em Oslo, foi considerada uma população seleta, com condições físicas saudáveis acima da população em geral. A coleta foi realizada do nariz e garganta. Eles concluíram que a incidência de flora patogênica foi constante, mas a incidência de resfriado foi maior no período do inverno. Os mesmos autores questionam a relação da flora bacteriana no resfriado comum, admitindo outro agente responsável e não a flora bacteriana. Por isso, eles consideraram improvável ser o Staphylococcus aureus o agente causador de sinusite aguda.

Karma et al.(1991), estudaram a sinusite crônica, e verificaram que o Staphylococcus aureus foi o germe mais freqüentemente isolado. Correlacionaram a cultura da nasofaringe com o seio maxilar, sendo observado $57,14 \%$ e a nasofaringe com a fossa nasal foi de $56,60 \%$.

Zelante et al.(1982, 1983), estudaram 130 pacientes sem queixas nasais ou bucais, e detectaram $20 \%$ de portadores de Staphylococcus aureus.

Kingdom et al.(1983), estudaram a flora nasal de estudantes de Medicina, e detectaram 29\% Staphylococcus aureus no Grupo A (estudantes sem exposição), 32\% no Grupo B (estudantes com 12 meses de exposição) e 32\% no Grupo C (estudantes com 36 meses de exposição). O número de Staphylococcus aureus resistente a 3 ou mais antibióticos foi de $9 \%$ em A, $0 \%$ em B e $55 \%$ em C. Com o Staphylococcus coagulase negativo obtiveram-se os seguintes resultados: no Grupo A 79\%, $97 \%$ de B e $94 \%$ de C. Portanto, não se verificaram diferenças significativas entre as cepas resistentes de coagulase negativa entre os 3 grupos. Os autores obtiveram também $10 \%$ das cepas de coagulase negativa resistente a três (3) ou mais antibióticos do grupo A, $16 \%$ de $\mathrm{B}$ e $15 \%$ de $\mathrm{C}$.

Lamikanra et al.(1985), ao estudarem a flora nasal de 548 estudantes nigerianos, identificaram $56,4 \%$ 
de Staphylococcus aureus. Os testes de sensibilidade mostraram que $94 \%$ dos organismos eram resistentes a penicilina.

Camargo et al.(1995), destacam a importância do estafilococo coagulase negativo. Nos EUA (Virgínea), nos últimos 7 anos a infeção por Staphylococcus epidermidis aumentou cerca de 4 vezes. Relatam 3 casos de infeções em recém nascidos da UTI neonatal. Entre pessoas que manipulavam essas crianças, $63 \%$ eram portadores de estafilococo coagulase negativo e o estudo pela análise do plasmídeo dos estafilococos permitiu identificar cepas idênticas em uma criança tanto na corrente sangüínea, quanto em esfregaços nasais. Enfatiza a importância da técnica do plasmídeo, que permite identificar as cepas causadoras da infeção.

Hollis et al.(1995), também salientam a infeção por Staphylococcus aureus, principalmente em pacientes em UTI neonatal. Utilizaram a técnica de identificação do agente com DNA, e conseguiram demonstrar que a infeção da criança foi por um Staphylococcus aureus que existia nas fossas nasais de dois irmãos e da mãe da criança.

Savolainen (1986), estudou a flora nasal em 404 indivíduos hígidos e verificou que pelo menos uma bactéria aeróbia se desenvolveu em cada cultura. A maior freqüência foi de anaeróbio em $51 \%$ das amostras. Obtiveram-se 62\% Staphylococcus epidermidis, 34\% de Staphylococcus aureus, e 47\% Propionibacterium acnes e $70 \%$ de difteróides. As bactérias patogênicas foram encontradas em $35 \%$, isto é o Haemophylus influenzae em 19\%, o Streptococcus pneumoniae em $13 \%$ e a Branhamella cattarahlis em $3 \%$. As bactérias patogênicas, que reconhecidamente causam sinusite, foram demonstradas em 5 a $7 \%$ dos indivíduos normais.

Segue a relação dos autores que estudaram a flora bacteriana nasal desde 1957 .

Quadro A - Relação de autores que estudaram a flora bacteriana nasal.

\begin{tabular}{|c|c|c|c|c|c|}
\hline AUTORES & ANO & Fonte de coleta de material & Staphylococcus aureus & Staphylococcus & Bac. Gram (--) \\
\hline HUTCHINSON & 1957 & Enfermeiras - fossa nasal & $56,90 \%$ & Coagulase negat & \\
\hline WHITE & 1961 & Pacientes Internados - fossa nasal & $37,11 \%$ & & \\
\hline MAXWELL & 1969 & Enfermeiras - fossa nasal & $62,00 \%$ & & \\
\hline RAHAL & 1970 & Médicos - enfermeiras nariz - garganta & & & $8,5 \%$ (hospital) \\
\hline ÇETIN & $1970-71$ & Estudantes universitários (vários cursos) & 7 a $20,50 \%$ & & \\
\hline DOIG & 1971 & Médicos - enfermeiras & 26 a $80,00 \%$ & & \\
\hline WINTHER & 1974 & Estudantes Ed. Física nariz - garganta & Etiologia sinusite aguda & & \\
\hline HECZKO & 1981 & Lab. Farmacêutico - fossa nasal & $11,60 \%$ & $30,90 \%$ & \\
\hline ZELANTE & $1982-83$ & Cav. Bucal Nariz - pacientes sem queixa & $20,00 \%$ & & \\
\hline KINGDOM & 1983 & Estudantes Medicina - fossa nasal & 29 a $32,00 \%$ & 79 a $97,00 \%$ & \\
\hline WEIL & 1984 & Farmácia - fossa nasal & & & $11,90 \%$ \\
\hline LAMIKANRA & 1985 & Estud. Universitário - fossa nasal & $56,40 \%$ & & \\
\hline SAVOLAIMEN & 1986 & Pessoas hígidas - fossa nasal & $34,00 \%$ & $62,00 \%$ & $70 \%$ difteróides \\
\hline CHOW & 1989 & Renal diálise - fossa nasal & $45,00 \%$ & & \\
\hline CEDERNA & 1990 & Internados em Asilo - fossa nasal & $38,20 \%$ & & \\
\hline GITTELMAN & 1991 & Pan sinusite & & & \\
\hline KARMA & 1991 & Sinusite crônica - Fossa nasal - Seio max. & $56,60 \%$ a $57,14 \%$ & & \\
\hline HOLLIS & 1995 & Neonatal - UTI - fossa nasal & Estudo com DNA & & \\
\hline CAMARGO & 1995 & Neonatal - UTI - plasmidio & & $63,00 \%$ & \\
\hline HESHIKI & $1995-96$ & Enfermagem Hospital - fossa nasal & $21,25 \%$ & $46,02 \%$ & $43,75 \%$ \\
\hline CVETNIC & 1996 & Laringectomizado (PO) - fossa nasal & $20,00 \%$ & $12,00 \%$ & $40,00 \%$ \\
\hline JOAQUIM & 1998 & Médicos residentes - fossa nasal & $31,80 \%$ & $81,80 \%$ & $34,00 \%$ \\
\hline HEL / C.C.I.H. & 2001 & Infeção Hospitalar (Out.2000-Jun. 2001) & $19,70 \%$ & & $80,30 \%$ \\
\hline $\mathrm{HU}$ & 2002 & Infecção Hosp. Jan.01 a Ago.01 Bol.n²0 & $19,40 \%$ & & $80,60 \%$ \\
\hline
\end{tabular}




\section{Materiais e Métodos}

O material foi coletado de fossa nasal dos médicos residentes, com atuação hospitalar e ambulatorial, no período de abril a setembro de 1998, escolhidos de forma aleatória envolvendo $68 \%$ dos residentes (88/ 129). A coleta foi realizada conforme técnica anteriormente descrita, (HESHIKI et al.,1996) introduzindo-se a zaragatoa em uma das fossas nasais para a realização de esfregaço em lâmina para a bacterioscopia. O tempo de permanência da zaragatoa em fossas nasais foi de aproximadamente 2 minutos. As amostras foram semeadas em ágar sangue e TSB (Triptic Soy Broth - Difco) incubadas a $35^{\circ} \mathrm{C}$ por 18 a 24 horas. As colônias desenvolvidas no ágar sangue foram submetidas à coloração de Gram e às provas bioquímicas específicas para bactérias Gram positivas e Gram negativas. Do caldo TSB (Triptic Soy Broth-Difco) foram repicados em ágar manitol sal vermelho de fenol (Merck) e McConkey (Merck), após incubação de um dia para outro a $35^{\circ} \mathrm{C}$. As colônias foram submetidas às provas bioquímicas específicas. Os germes identificados foram submetidos ao teste de sensibilidade (antibiograma).

\section{Resultados}

Do material coletado da fossa nasal de 88 médicos residentes, foram isolados 21 espécies de bactérias, totalizando 181 cepas. Os microorganismos mais freqüentes foram: Estafilococos coagulase negativo $(39,23 \%)$, Staphylococcus aureus $(17,68 \%)$ e bactérias Gram neg. (31,91\%), e estão relatados nas (Tabelas 1 e 2). Todos os residentes apresentaram crescimento de pelo menos uma bactéria (Tabela 3); em $94.32 \%$ dos residentes apresentaram de 1 a 3 bactérias. A média de cepas identificadas por residente foi de 2,05\% (181/88).

Os microorganismos foram submetidos aos testes de sensibilidade e as cepas resistentes estão ilustradas na Tabela 4. Penicilina 29,48\%, Eritromicina 19,52\%, Tetraciclina 11,95\% Cloranfenicol 8,76\%, Cefalosporina $7,17 \%$.

Dos 88 médicos residentes, 32\% (29/88) possuíam resistência bacteriana para mais de 3 anti- bióticos em pelo menos 1 microorganismo. Estafilococos coagulase negativo deu 30,98\% (22/

71) resistentes a mais de 3 antibióticos; Staphylococcus aureus, 15,62\% (5/32) e das bactérias Gram negativas 9,3\% (4/43).

Tabela 1 - Espécie de bactérias identificadas em 88 residentes.

\begin{tabular}{|l|c|c|}
\hline \multicolumn{1}{|c|}{ Bactérias } & $\mathrm{N}^{\mathbf{0}}$ de cepas & \% total de cepas \\
\hline 01 Staphylococcus coagulase Negativa & 71 & $\mathbf{3 9 , 2 3} \%$ \\
\hline 02 Staphylococcus aureus & 32 & $\mathbf{1 7 , 6 8}$ \\
\hline 03 Staphylococcus saprophyticus & 2 & 01,10 \\
\hline 04 Streptococcus viridans & 4 & 02,21 \\
\hline 05 Micrococcus sp & 2 & 01,10 \\
\hline 06 Acinetobacter baumannii & 2 & 01,10 \\
\hline 07 Xanthomonas maltophilia & 1 & 00,55 \\
\hline 08 T. ptyseos & 1 & 00,55 \\
\hline 09 Morganella (Branhamella) morgani & 1 & 00,55 \\
Sub total & $(116)$ & $\mathbf{6 4 . 0 9 \%}$ \\
\hline
\end{tabular}

Tabela 2-Bactérias Gram Negativas identificadas em 88 residentes.

\begin{tabular}{|c|c|c|}
\hline $01 \quad$ - Bacillus sp (anaerobios) & 19 & $\mathbf{1 0 , 5 0}$ \\
\hline $02 \quad$ - Pseudomonas $\mathrm{sp}$ & 9 & 04,97 \\
\hline - Pseudomonas stutzezi & 4 & 02,28 \\
\hline - Pseudomonas aeruginosa & 1 & $\mathbf{0 0 , 5 0}$ \\
\hline 05 - Proteus mirabilis & 4 & $\mathbf{0 2 , 2 8}$ \\
\hline 06 - Enterobacter sp & 8 & 04,57 \\
\hline 07 - Enterococcus aerogenes & 7 & $\mathbf{0 3 , 8 7}$ \\
\hline 08 - Escherichia coli & 7 & $\mathbf{0 3 , 8 7}$ \\
\hline 09 - Enterococcus sp & 2 & 01,10 \\
\hline 10 - Klebsiella pneumoniae & 2 & 01,10 \\
\hline 11 - Klebsiella oxytoca & 1 & 00,50 \\
\hline 12 - Klebsiella ozonae & 1 & 00,50 \\
\hline Sub total & $(65)$ & $31.91 \%$ \\
\hline $\begin{array}{c}\text { Total } 21(9+12) \text { espécies de } \\
\text { bactérias }\end{array}$ & $116+65=181$ & $100,00 \%$ \\
\hline
\end{tabular}


Tabela 3 - Número de bactérias identificadas em 88 residentes.

\begin{tabular}{|c|c|c|c|}
\hline $\mathrm{N}^{\circ}$ Bactérias desenvolvidas & $\mathrm{N}^{\circ} \quad$ Residentes & $\%$ & \\
\hline 0 & 00 & 00 & \\
\hline 1 bactéria & 31 & 35,23 & \\
\hline 2 Bactérias & 35 & 39,77 & \\
\hline 3 Bactérias & 17 & 19,32 & $94,32 \% *$ \\
\hline 4 Bactérias & 03 & 3,40 & \\
\hline 5 Bactérias & 02 & 2,27 & \\
\hline TOTAL & 88 & $100.00 \%$ & \\
\hline
\end{tabular}

* Soma de residentes com $(1,2,3)$ Bactérias 35,23 + 39,77 $+19,32=94,32 \%$.

(\%) Indica o percentual de residentes em relação ao total de 88 residentes

Proporção do total de bactérias identificadas entre os residentes $=181: 88=2.05$

\section{Discussão}

O estudo da flora nasal dos médicos residentes permitiu identificar 21 espécies de bactérias, totalizando 181 cepas. Desse total 31,91\% foram bactérias Gram (一). As cepas mais freqüentes (Tabela 1) foram:

1- Staphylococcus coagulase negativa $(39,23 \%)$. Os autores consultados mostram índices que variam de 12\% (CVETNIC et al.,.1996), 63\% (CaMARGO, 1995) a 97\% (KINGDOM et al.,.1983).

2- Staphylococcus aureus $(17,68 \%)$ a variação entre os autores consultados é de 11,6\% (HECZCO, 1981), 56,9\% (HUTCHINSON, 1957), 62\% (MAXWELL, 1969), até 80\% (DOIG, 1971).

Entre os Gram negativos (Tabela 2) foram detectadas 12 espécies de bactérias, o que corresponde a $31,91 \%$. Os mais frequientes foram Bacillus sp (10,5\%), as Pseudomonas (7,55 \%), Enterobacter sp (4,57\%), Enterococcus aerogenes (3,87\%), Escherichia coli $(3,87)$, Proteus mirabilis $(2,28 \%)$. Os autores consultados apresentam índices variáveis de 8,5\% (RAHAL,1970), 11,9\% (WEIL,1984), ATÉ 40\% (CVETNIC, 1996).
A Tabela 3 mostra que todos os residentes apresentaram pelo menos 1 bactéria e que a maioria $(94,32 \%)$ apresentou 1, 2 ou 3 bactérias.

Quanto à resistência bacteriana, foi possível realizar testes de sensibilidade para 13 tipos de antibióticos disponíveis (Tabela 4). A resistência foi acima de $10 \%$ para penicilina, eritromicina e tetraciclina, e abaixo de $2 \%$ para ciprofloxacin, oxacilina, rifamicina e cefepime.

Tabela 4 - Teste de sensibilidade aos antibióticos.

\begin{tabular}{|l|c|c|}
\hline Antimicrobiano & $\mathrm{N}^{\mathbf{0}}$ cepas resistentes & $\%$ \\
\hline 1 - Penicilina & 74 & 29,48 \\
\hline 2 - Eritromicina & 49 & 19,52 \\
3 - Tetraciclina & 30 & 11,95 \\
4 - Cloranfenicol & 22 & 8,76 \\
\hline 5 - Cefalosporina & 18 & 7,17 \\
\hline 6 - Sulfametoxazol-Trimetropin & 15 & 5,98 \\
\hline 7 - Clindamicina & 14 & 5,58 \\
\hline 8 - Gentamicina & 8 & 3,19 \\
\hline 9 - Ampicilina & 8 & 1,19 \\
\hline 10 - Ciprofloxacina & 3 & 1,19 \\
\hline 11 - Oxacilina & 3 & 1,19 \\
\hline 12 - Rifampicina & 3 & \\
\hline 13 - Cefepime & & \\
\hline Total & $3900 \%$ \\
\hline
\end{tabular}

\section{Conclusão}

Pode-se concluír que 94\% dos médicos residentes de Hospitais Universitários de Londrina são portadores de 1 a 3 bactérias nas fossas nasais. Das bactérias identificadas as mais freqüentes foram o Staphylococcus coagulase negativa (39\%), Staphylococcus aureus (17\%), e as bactérias Gram negativas (32\%). 
Foram identificadas e testadas 21 espécies de bactérias para 13 antibióticos. Os testes de sensibilidade aos antibióticos disponíveis demonstram que são resistentes à penicilina (29\%), eritromicina (19\%), terramicina $(12 \%)$ e cloranfenicol $(9 \%)$. A resistência abaixo de $2 \%$ foi para ciprofloxacin, oxacilina, rifampicina e a cefepime. O Staphylococcus coagulase negativo, considerado normal para a flora nasal, mostrou-se de resistência elevada.

\section{Referências}

BARTH, J.H., (England) Nasal carriage of Staphylococci and Streptococci. Int. J. Dermatol., Oxford, v.26,n.1, p.246, 1987.

BÉRÉZIN,E.B.; GUILLOU,M.J.L.; VIEU, J.F. Epidemiology of nosocomial infections due to Acinetobacter calcoacetius. J. Hosp. Infection, London, v.10, p.105-113, 1987.

CAMARGO, L.F.A., et al. Epidemiological investigation of an outbreak of coagulase negative Staphylococcus primary bacterium in a newborn intensive care unit. Infect. Control. Hosp. Epidemiol., Thorofare, v.16, n.10, p. 5956, 1995.

CEDERNA,J.E. et al., (Ann Arbor) Staphylococcus aureus nasal colonization in a nursing home: eradication with mupirocin. Infect. Control. Hosp. Epidemiol., Thorofare, v.11,n.1, p.13-16,1990.

CETIN E.T., et al.Investigations on aerobic oral and nasal flora of University students. Pathology Microbiol., Basel, v.37, n.3, p. 185-93, 1971.

CHOW, J.W., YU, V.L. e col. Staphylococcus aureus nasal carriage in hemodialysis patients. Its role in infection and approaches to prophylaxis. Arch. Internat. Med., Chicago, v. 149, n.6, p.1258-1262, Jun.1989.

CVETNIC,W.G., et al. Comparison of continuous negative pressure and nasal positive airway. Eur. Respir. J., Sheffield,v.9, p.1531-44, 1996.

DOIG, C. M. Nasal carriage of Staphylococcus aureus in a general surgical unit Brit. J. Surgery, Oxford, v.58,n.2, p.113/116, 1971.

GITTELMAN P. D., et al.(New Haven)- Staphylococcus aureus nasal carriage in patients with rhino sinusitis. The Laryngoscope, St. Louis, v.101, n.7, p.733-737, 1991.

HECZKO, P.B., et al. (Poland). Quantitative studies of the flora of the nasal vestibule in relation to nasal carriage of Staphylococcus aureus. J. Med. Microbiol., London, v.14, n.3, p.233-241, 1981.
HESHIKI, RE., et al. Estudo da flora bacteriana nasal: estudo preliminar em equipe de enfermagem. Semina, Londrina, v. 17, n.2, p. 191-196, Jun 1996.

HESHIKI, Z., QUESADA, R. M.M , HESHIKI, R. E. - La flora bacteriana nasal - An. Otorrinolaringol., Peru, v.6, n.3, p.253-262, 1999.

HOLLIS R.J., et al. Familial carriage of methicillin-resistant Staphylococcus aureus and subsequent infection in a premature neonate. Clinical Infect. Diseases, v.21, n.2, p.328-332, 1995.

HUTCHISON, J.G.P.; GREEN, C.A.; GRIMSON, T.A. Nasal carriage of Staphylococcus aureus in nurses. J. Clin. Path., London, v.10, n.1, p.92-95, 1957.

JOHANSON,W.G.; PIERCE, A.K. SANFORD, J.P. Changing pharyngeal bacterial flora of hospitalized patients. New Eng. J. Med., Boston, v.281,n.21, p.11371140, 1969.

KARMA, P., et al. The comparative efficacy and safety of clarithromycin and amoxycillin in the treatment of outpatients with acute maxillary sinusitis. J. Antimicrob. Chemotherapy, London, v. 27, suppl. A, p. 83-90, fev. 1991.

KINGDOM, et al. (Dublin). Staphylococcus aureus nasal carriage in medical students with varying clinical exposure J. Hosp.Infection, London, v.4, n.1, p.75-79, 1983.

LAMIKANRA, A. et al. Nasal carriage of Staphylococcus aureus in a population of healthy Nigerian students. J.Med. Microbiol., London, v.19, n.2, p.211-216, 1985.

MAXWELL J.G, et al. Long-term study of nasal staphylococci among hospital personnel. Amer. J.Surg., New York, v.118, n.6, p.849-854, 1969.

O'GRADY, F.; WITTSTADT, F. B. Nasal carriage of Staphylococcus pyogenes. Am.J. Hyg.,Baltimore, v.77, n.2,p.187-194,1963.

RAHAL Jr. J.J.; MEADE, R.H.; BUMP, C.M.; REINAUER, A.J. Upper Respiratory Tract Carriage of Gram Negative Enteric Bacilli by Hospital Personnel. J.Am. Med. Assoc., Chicago, v.214, p.754-756,1970.

RODER B.L., et al. No difference in enterotoxin production among Staphylococcus aureus strains isolated from blood compared with stains isolated from healthy carriers. J.Med. Microbiol., Edinburg, v. 42, n.1, p. 43-47, Jan. 1995.

SAVOLAINEN, S., YLIKOSKI, J., JOUSIMLES S. - The bacterial flora of the nasal cavity in healthy young men. Rhinology, Utrecht, v. 24, p.249-255, 1986.

WEIL, D.C.; ARNOW, P.M.(Chicago) Prevalence of gram negative bacilli in nares and on hands of pharmacy personnel: lack of effect of occupational exposure to antibiotics. J. of Clin. Microbiol., Washington, v.20, n.5, p.933-935,1984. 
WHITE, A. Relation between quantitative nasal cultures and dissemination of staphylococci. J.Lab. \& Clin. Med. , St. Louis, v.58, n.2, p. 273-277, 1961.

WINTHER, F.O. et al.(Oslo) Pathogenic bacterial flora in the upper respiratory tract of health students. Prevalence and relationship to nasopharyngeal inflammatory symptoms. J. Laryng. Otol., Ashford,v.88, p. 407-412,1974.
ZELANTE,F. et al. Staphylococcus aureus na boca e no nariz de indivíduos sãos. Verificação de identidade entre as cepas isoladas. Rev. Saúde Publ., São Paulo, v.16, n.2, p.92-96, 1982.

ZELANTE, F. et al. Observações sobre o padrão fágico de cepas de Staphylococcus aureus isoladas da boca e do nariz de indivíduos sãos. Rev. Saúde Publ., São Paulo, v.17, n.2, p.123-129,1983. 